\title{
Research on Consistency between Mental Model and Information Visualization and Practice in primary school Sign System Design
}

\author{
Chunfu $\mathrm{Li}^{1, \mathrm{a}}$, Luying Chen ${ }^{2, \mathrm{~b}}$ \\ ${ }^{1}$ Huazhong University of Science and Technology, Wuhan, P.R. China, 430074 \\ ${ }^{2}$ Huazhong University of Science and Technology, Wuhan, P.R. China, 430074 \\ a504971652@qq.com, bchenluying2014@163.com,
}

Keywords: sign \& guide system design, information visualization, mental model

\begin{abstract}
With the expansion of the size of the primary school building, campus space are becoming increasingly complex. In order to meet the needs of students learning and life, primary school identification system emerge at the right moment. As an important part of campus environment and culture, school identification system is the material carrier of campus information and the external representation of the campus environment. From the relationship between the user's mental model and campus information visualization, the research focus on the relationship of information delivery performance between students mental model and "text"、 "graphics" and "text with graphic" the three types of information visualization methods, and mental models mapping and reflecting in identification systems, so as to explore that there exists consistency between the user's mental model and the types of information visualization methods "text with graphic".
\end{abstract}

\section{Introduction}

Nowadays, owing to the increasingly competition for knowledge and the talent, education all around the world has been an unprecedented degree of attention. Since the reform and opening up, to change the lack of flexible impasse, China's basic education has developed the local, diversified school system, in line with improving the quality of school education, primary school environment should be designed to meet the quantity, function and other basic needs of the premise by seeking a higher platform.

In the past, due to teaching models and financial constraints and other factors, people focus on the improvement of the indoor environment and facilities to improve the quality of the school environment, and think outdoor space is the "negative space" after the completion of construction, ignoring the outdoor campus environments shape. With the country supporting primary education and assistance in recent years, both in the quality of education and school environment on campus have a considerable development. But now many primary schools are facing many problems that the various campus buildings, the complex environment of space and crisscrossing roads, these will undoubtedly bring some inconveniences to younger students on the daily life.

However, as a special population, there are obvious differences between pupils and adults in awareness and sensitivity. If the design of school campus identification system does not take the primary groups of students into account, and then the effect is often unattainable, identification system with indefinite information visualization may lead students unable to find the destination, even plays the reverse effect. In this paper, through experimental tests, we research the usability test results matching the user's mental model and analyze information for different pupils, visual way to design a logo system, improving usability of primary school logo design from the source.

\section{Literature References}

Information visualization .At the beginning, information visualization has not been clearly defined, it gradually evolves and form from other disciplines, its origins can be dated back to the late eighteenth century, when people use the abstract visual representation way to express data, in this way continuously to explore the mysteries and unknown information data model, this exploration 
gives birth to data graphics. "Information Visualization" the term firstly appeared in G.Roh-ertson, S.Card and J.Mackinlay 's paper in 1989, today information visualization has become a scientific visualization research. As shown in Figure 1 is the evolution of information visualization methods, from simple text in the past slowly adding the graphics and tables. In order to make the information more quickly perceived and accepted, some designers introduce a form of "dynamic interaction" to break the information visualization graphics performance, bringing a new, more intuitive form of expression, and this form is mainly used in network information design.
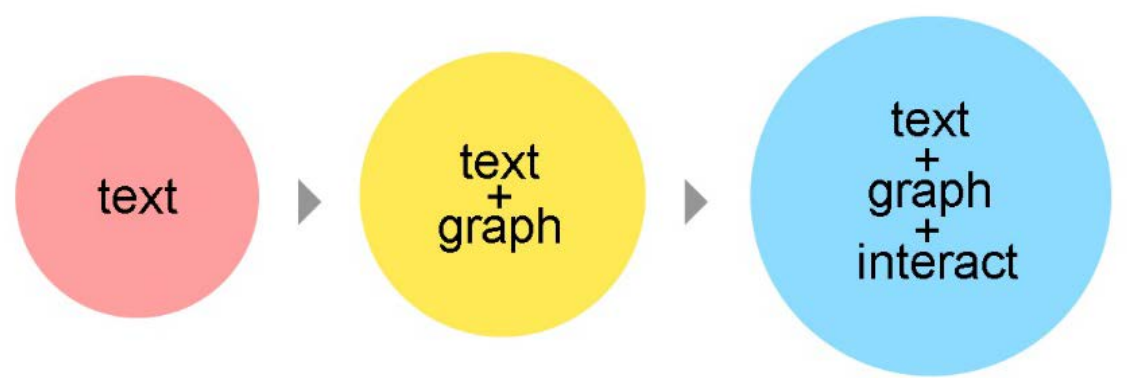

Fig. 1 The evolution of information and data presentation

Mental model .Mental model is a concept derived from cognitive psychology. Firstly proposed in 1943 by Kenneth Craik, Craik think mental model is used to explain the cognitive process of the inherent individual in the real world [1]. Psychologists Johnson-Laird PN interprets mental model as a simpler world in our mind when people have an understanding of the objective world [2]. It is an dynamics internal representation for human to outside world or event. Because mental model has a strong internal resistance and is difficult to visualize, the majority of research has emerged as a holistic overview of the features [3]. Research on the mental model natural phenomena by Vosniadou et al [4], although mental model is an internal representation, but this characterization is not completely simulate the outside world for the event, it has simpler structure compared with external objects, and this simulation object is not entirely correspond to its characterization [5]. Indi • Young [6] believes the mental model is a powerful tool for researchers or designers to understand the users' motives, thought processes, emotional outlook and philosophy, and emphasizes mental model brings the root causes together that people engage in something or tasks unrelated to their tools; Mental models only focus on relatively stable patterns of behavior for specific populations, not only represent the kind of atypical certain scenarios model.

For the relationship between information visualization and mental models, Robert Spence from the British point out in the "Information Visualization: interaction design": "Visualization is to establish mental models or mental image for something, therefore, visualization is purely human cognitive activities unrelated with computer [7]."'From this we can find that the most central point of information visualization is the visualization. By understanding the concept of visualization, we can find the essence of information visualization is actually a process to establish mental model by the design the information data.

The consistency between mental model and information visualization. Currently the consistency problems of identity-oriented design focus on the "repetitive" and "similarity". In fact, when students understand the identification system and complete the oriented tasks, there is another important consistency: the consistency between user's existing mental model and information visualization, which is caused by different visual form (text, graphics, text with graphics) and feedback caused, from the beginning to the end of the whole cognitive process.

For users who are familiar with campus identification system, during browsing, the situation that recognize and search visual is more than the situation that recalls. That means that the role of mental models is not important when users have been familiar with the mark. For a new student, mental models often play the role of guide and forecasting, as identity-oriented design is achieved by plane visual visualization. Visualization of this information will have on the real space-oriented capabilities. As shown in figure 2, if there exists new some consistency between identity-oriented design of graphics, text and users' mental model, then the existing mental models will be applied by users and 
would have the effect of strengthening deepening. If there are inconsistency between the content of information visualization and the user's mental model, then there will be confusion, misjudgment phenomenon, and will modify their original passive mental model to adapt to the reality of things.

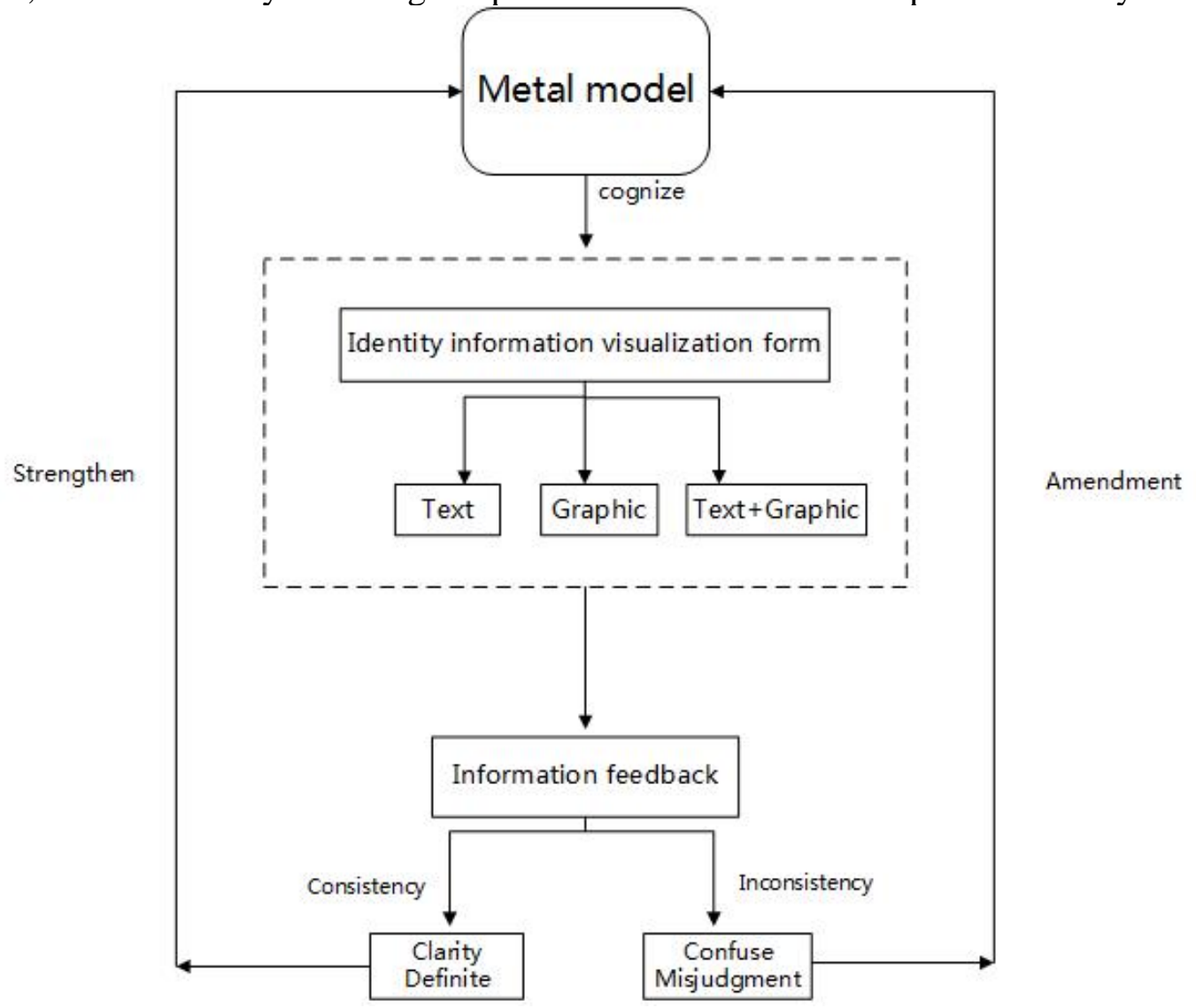

Figure 2 The operation way of mental model

\section{Usability evaluation}

Usability is generally expressed as "user-friendly", "intuitive", "easy to use", "do not need long-term training," "no brainer" and so on. Specifically it refers that the effectiveness, efficiency and satisfaction of a product when used for specific purposes in a particular environment scenario, a particular user.

Nielsen believes that usability can be divided into the following five dimensions [8]. When the product have reached a higher level in the following five dimensions, it can be evaluated as high availability.

(1) Learnability: the system is easy to learn and master.

(2) Efficiency: the user can efficiently use the system.

(3) Memorability: the system should be easy to remember, after able to use the system, even if there is a long time not to use the system, users can quickly grasp the method of operation when using the system again, instead of re-learning.

(4) Errors: The system should reduce errors as much as possible, and have some ability to correct errors and recovery capabilities, and will not appear irreversible system error.

\section{Consistency test}

In this part, we explore reasonable consistency between the mental model and information visualization by experiment, using a statistical experimental approach. The experiment is to clearly know their needs by statistics on the basic stages of primary identification card but different forms of visual recognition performance. Experimental hypothesis: if the existing mental model which is 
consistent with a visual form of signage content, then the information transfer performance is higher than the inconsistent one.

Respondent and Survey unit. Respondents are students from Huazhong University of Science Preparatory School. It is divided into three groups according to the survey grade: the first group of first grade students, called as the "first-year group", the second group of third grade students, called as the "three-year", and the third group was in the sixth grade students, known as the "six-year group." The number of respondents in each group is 10; the proportion of men and women in each group is 1 : 1 .

Materials and equipment. The experimental tests are conducted on all tablet PC. The experimental materials are the basic marks for elementary school in recent years. There are respectively 30 "Text" "Graphics", "graphic with the words "category signage.

Experimental procedures. Since the object of this investigation are pupils, whose understanding ability is worse than adults, in order to ensure the reliability of the experimental survey data, therefore it needs to introduce the test process before the survey, only when respondents fully understand and appreciate the experiment, we can make real test.

There are 10 multiple choice tests with the same questions in 3 rounds in this experimental survey. When respondents look at the tests, click "Next", enter the answer selection. The answer of first round is 3 "text" visual form of identification card, the answer of second round is 3 "Graphic" visual form of identification card, and the answer of third round is 3 "text with graphics" visual form of identification card. Each group has a correct answer, based on their understanding of the subject, they can choose an answer they think is correct, click it and then enter the next page. As shown in Figure 3 is the specific steps.

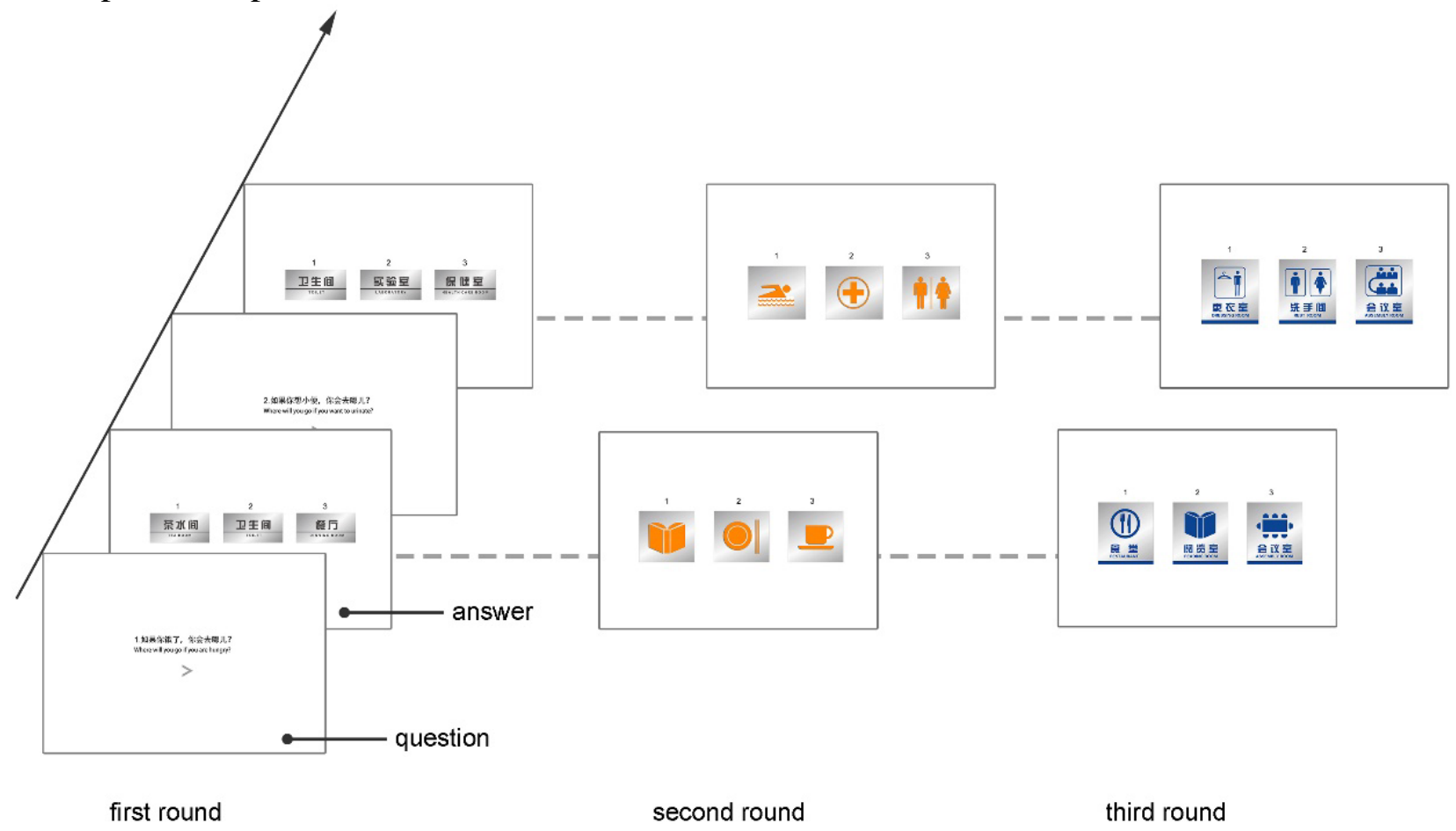

Fig. 3 Experimental process

\section{Results and discussion}

To sort, count and analyze experimental tests results and consistency and correctness between information visualization and mental model can be verified.

The results are counted and analyzed from "grade one" "grade three"," grade six" three group data. To make the results of the evaluation of the availability of more intuitive, the mean reaction time of $\mathrm{M}$, the accuracy of the answer to Q are used as the evaluation index. As experiments are conducted on a tablet PC, we can easily get the elapsed time .As shown in Table 1 is the statistical results. 
Table 1 Statistical results

\begin{tabular}{l|lll}
\hline \multicolumn{1}{l}{ GROUPS } & VISUAL FORM & $\begin{array}{l}\text { THE MEAN } \\
\text { REACTION TIME } \\
\text { M/MS }\end{array}$ & $\begin{array}{l}\text { THE ACCURACY } \\
\text { Q/\% }\end{array}$ \\
& & 5418 & 82 \\
\hline \multirow{3}{*}{ GRADE ONE } & Text & 4517 & 85 \\
& Graphic & 4232 & 85 \\
\hline \multirow{3}{*}{ GRADE THREE } & Text+ Graphic & 3823 & 96 \\
& Text & 4128 & 95 \\
& Graphic & 3616 & 98 \\
\hline \multirow{3}{*}{ GRADE SIX } & Text+ Graphic & 2465 & 100 \\
& Text & 3154 & 99 \\
& Graphic & 2254 & 100
\end{tabular}

We can firstly analyze the three groups' experimental results and then analyze the data between different groups, which can better reflect the consistency of the different forms of visual and mental models.

Grade one.From statistical results of group one, for visualization in the form of text, the mean reaction time is longest among the three groups, while its answer accuracy rate is the lowest, and the second group results are close to the third group in reaction time and accuracy. It shows that for low-grade students, its poor character recognition ability, limited by the understanding of the text, so they choose the answer mainly by memory with the usual experience, word length, but for the graphics, their choice is consistent with their existing mental models. So for the low-grade students, the graphics visual form match with their existing mental model.

Grade three.From statistical results of second group, the results for the text and graphic are close in reaction time and accuracy, the answer for the form of text plus graphics in reaction time and accuracy is better, while difference between these three groups is not obvious. Compared with group one, third-grade students already have character recognition capability, grasped of common words basically, so it speeds a faster response time compared the group one in text and the accuracy has significantly improved, also shows that the consistency between the visual form of text and already existing mental model is better, while compared to group one, the reaction time for the graphics declines and the accuracy has increased, indicating that its graphics cognitive ability has been strengthened compared to first-grade students, and the text with graphical visualization is best, with the most consistent with mental model.

Grade six.From statistical results of third group, the accuracy of the three forms of visual is close, and in the reaction time, visual graphical form is relatively slowest, text and text plus visual graphical the two form is close, corresponding to the first grade and third grade student group, the accuracy rate and reaction time have been significantly improved, indicating that for the sixth grade students, their existing mental model is corresponded to form of text, and for the graphic form, the reaction time is slower, indicating that it takes a longer time for graphic form to test form, the sensitivity of the graphics has dropped with increase of age. Therefore, for sixth grade students in the group, the form of text visualization can better meet their mental models.

Results of the above analysis, it mainly consider the factors different groups and different grades visual form affecting results, there are still other factors which influence the results , especially the gender factor, after all, men and women's way of thinking are different. Therefore, experimental data are conducted the main effect analysis, as shown in Table 2 is the result. $\mathrm{F}$ is the value of F-test, $\mathrm{P}$-value is significance test value, $\mathrm{SS}$ is the variance and MS is mean square. It shows that the value of MS 512672 for the visual form, which is much larger than gender factors MS 8066.666667, indicating differences in the average response time caused by visual form are much larger than caused by gender factors. At the same time, the $\mathrm{F}=16.5075<18.5128=\mathrm{F}$ crit for the gender factor, indicating that the sex factor has no significant impact on the results, and the P-value $=0.05557>0.05$, 
explaining that the gender factor has no significant difference at the 0.05 level for the reaction time. For the visual form, $\mathrm{F}=1049.124>19=\mathrm{F}$ crit, while $\mathrm{P}$-value $=0.00095<0.05$, the results illustrate that visual forms have a significant impact on the results, while the reaction time has significant difference at the 0.05 level.

At this point, it is sure that the factors influencing reaction time and accuracy of the answer come from age factors and visualization of different forms. As shown in experiments, when the consistency between visual form and mental model is high, the corresponding reaction time and accuracy significantly are better than lower one, therefore, in primary identity-oriented design, considering different consistency for different grades students' existing mental models with the different visual forms, visualization form of comprehensive text with graphics can achieve the purpose of the information transmission on the maximum range.

Table 2 Two way ANOVA analysis for visual form and gender factors

\begin{tabular}{lrrrrr}
\hline Differences source & \multicolumn{1}{c}{ SS } & \multicolumn{1}{c}{ MS } & F & P-value & \multicolumn{1}{c}{ F crit } \\
\hline Gender & 8066.6667 & 8066.666667 & 16.50750341 & 0.055576514 & 18.512821 \\
Visual form & 1025344 & 512672 & 1049.124147 & 0.000952268 & 19 \\
Error & 977.33333 & 488.6666667 & & & \\
Total & 1034388 & & & & \\
\hline
\end{tabular}

\section{Summary}

In this paper, we take the elementary school logo-oriented design as research object, starting from perspective of consistency between the mental model and information visualization, discussing usability issues in identity-oriented design. Experimental studies suggest that consistency between mental model and information visualization are important factors affecting the availability of user awareness. The higher consistency mental models and information visualization is, the higher the information transfer performance and availability are; On this basis, the suggest is proposed for primary school logo-oriented design based on consistency between the user's mental model and information visualization, that is the visual form of comprehensive text and graphics can convey information best. It will offer some help to primary school logo-oriented design in future.

\section{References}

[1]Kenneth Craik. The nature of explanation Cambridge-Cambridge University Press, 1943

[2]Johnson-Laird PN. Mental modes: towards a cognitive science of language, inference and consciousness. Cambridge: Harvard University Press. 1983

[3]SASSE M A. Eliciting and describing users' models of computer systems [D]. Birmingham: University of Birmingham, 1997.

[4]VOSNIADOU S, BREWERW F. Mental models of the day/night cycle [J].Cognitive Science, 1994, 18: 123-183.

[5]JOHNSON-LAIRD P N. Mental models: towards a cognitive science of language, inference and consciousness [M]. Cambridge: Harvard University Press, 1983.

[6]Indi Young. Mental Models: Aligning Design Strategy with Human Behavior. New York: Rosenfeld Media, 2008.

[7]Robert Spence, translated by Chen Yaqian, "information visualization": interaction design, mechanical industry press, 2011.

[8] Nielsen, J, translated by Liu Zhengjie. Usability engineering. Mechanical Industry Press, 2004 